Kärner, Tobias; Reinke, Hannes; Frim, Anja; Heinrichs, Karin

\title{
Innere Differenzierung im Unterricht mit jugendlichen Asylsuchenden und Geflüchteten aus der Sicht von Lehrpersonen
}

Wittmann, Evelyn [Hrsg.]; Frommberger, Dietmar [Hrsg.]; Weyland, Ulrike [Hrsg.]: Jahrbuch der berufs- und wirtschaftspädagogischen Forschung 2019. Opladen ; Berlin ; Toronto : Verlag Barbara Budrich 2019, S. 59-74. (Schriftenreihe der Sektion Berufs- und Wirtschaftspädagogik der Deutschen Gesellschaft für Erziehungswissenschaft (DGfE))

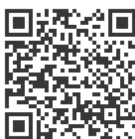

Quellenangabe/ Reference:

Kärner, Tobias; Reinke, Hannes; Frim, Anja; Heinrichs, Karin: Innere Differenzierung im Unterricht mit jugendlichen Asylsuchenden und Geflüchteten aus der Sicht von Lehrpersonen - In: Wittmann, Eveline [Hrsg.]; Frommberger, Dietmar [Hrsg.]; Weyland, Ulrike [Hrsg.]: Jahrbuch der berufs- und wirtschaftspädagogischen Forschung 2019. Opladen ; Berlin ; Toronto : Verlag Barbara Budrich 2019 , S. 59-74 - URN: urn:nbn:de:0111-pedocs-184363 - DOI: 10.25656/01:18436

https://nbn-resolving.org/urn:nbn:de:0111-pedocs-184363

https://doi.org/10.25656/01:18436

in Kooperation mit / in cooperation with:

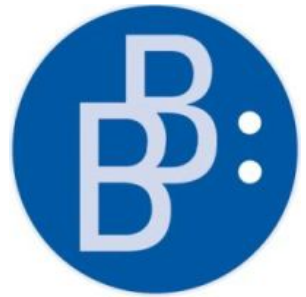

https://www.budrich.de

\section{Nutzungsbedingungen}

Dieses Dokument steht unter folgender Creative Commons-Lizenz: http://creativecommons.org/licenses/by-sa/4.0/deed.de - Sie dürfen das Werk bzw. den Inhalt vervielfältigen, verbreiten und öffentlich zugänglich machen sowie Abwandlungen und Bearbeitungen des Werkes bzw. Inhaltes anfertigen, solange sie den Namen des Autors/Rechteinhabers in der von ihm festgelegten Weise nennen und die daraufhin neu entstandenen Werke bzw. Inhalte nur unter Verwendung von Lizenzbedingungen weitergeben, die mit denen dieses Lizenzvertrags identisch, vergleichbar oder kompatibel sind.

Mit der Verwendung dieses Dokuments erkennen Sie die Nutzungsbedingungen an.

\section{Terms of use}

This document is published under following Creative Commons-License: http://creativecommons.org/licenses/by-sa/4.0/deed.en - You may copy distribute and transmit, adapt or exhibit the work or its contents in public and alter, transform, or change this work as long as you attribute the work in the manner specified by the author or licensor. New resulting works or contents must be distributed pursuant to this license or an identical or comparable license.

By using this particular document, you accept the above-stated conditions of use.

\section{Kontakt / Contact:}

peDOCS

DIPF | Leibniz-Institut für Bildungsforschung und Bildungsinformation Informationszentrum (IZ) Bildung

E-Mail: pedocs@dipf.de

Internet: www.pedocs.de

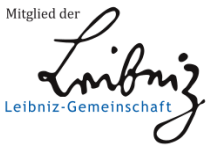




\section{Jahrbuch der berufs- und wirtschaftspädagogischen Forschung 2019}

Eveline Wittmann, Dietmar Frommberger, Ulrike Weyland (Hrsg.)

\section{DGfE}


Jahrbuch der berufs- und

wirtschaftspädagogischen Forschung 2019 


\section{Schriftenreihe der Sektion \\ Berufs- und Wirtschaftspädagogik der Deutschen Gesellschaft für \\ Erziehungswissenschaft (DGfE)}


Eveline Wittmann

Dietmar Frommberger

Ulrike Weyland (Hrsg.)

Jahrbuch der berufs- und

wirtschaftspädagogischen

Forschung 2019

Verlag Barbara Budrich

Opladen • Berlin • Toronto 2019 
Bibliografische Information der Deutschen Nationalbibliothek

Die Deutsche Nationalbibliothek verzeichnet diese Publikation in der Deutschen Nationalbibliografie; detaillierte bibliografische Daten sind im Internet über

http://dnb.d-nb.de abrufbar.

(C2019 Dieses Werk ist bei der Ve rlag Barbara Budrich GmbH erschienen und steht unter der Creative Commons Lizenz Attribution-ShareAlike 4.0 International (CC BY-SA 4.0): https://creativecommons.org/licenses/by-sa/4.0/.

Diese Lizenz erlaubt die Verbreitung, Speicherung, Vervielfältigung und Bearbeitung bei Verwendung der gleichen CC-BY-SA 4.0-Lizenz und unter Angabe der UrheberInnen, Rechte, Änderungen und verwendeten Lizenz. www.budrich.de

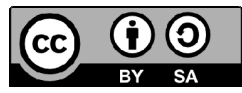

Dieses Buch steht im Open-Access-Bereich der Verlagsseite zum kostenlosen Download bereit (https://doi.org/10.3224/84742330).

Eine kostenpflichtige Druckversion (Print on Demand) kann über den Verlag bezogen werden. Die Seitenzahlen in der Druck- und Onlineversion sind identisch.

$$
\begin{array}{ll}
\text { ISBN } & 978-3-8474-2330-0 \text { (Paperback) } \\
\text { eISBN } & 978-3-8474-1351-6 \text { (eBook) } \\
\text { DOI } & 10.3224 / 84742330
\end{array}
$$

Umschlaggestaltung: Bettina Lehfeldt, Kleinmachnow - www.lehfeldtgraphic.de Druck: Books on Demand GmbH, Norderstedt Printed in Europe 


\section{Inhaltsverzeichnis}

Aktuelle theoretische und empirische Perspektiven der Berufs- und Wirtschaftspädagogik

\section{Teil I: Berufsbildungs-, organisations- und professionstheoretische Perspektive}

Franz Kaiser und Thilo J. Ketschau

Die Perspektive kritisch-emanzipatorischer Berufsbildungstheorie als

Widerspruchsbestimmung von Emanzipation und Herrschaft

Marc Egloffstein, Tobias Heilig und Dirk Ifenthaler

Entwicklung eines Reifegradmodells der Digitalisierung für Bildungsorganisationen

Karin Reiber, Ulrike Weyland und Eveline Wittmann

Professionalisierung des schulischen Bildungspersonals in den Gesundheits- und Pflegeberufen - Zwischenfazit eines berufs- und wirtschaftspädagogischen Sonderweges

\section{Teil II: Berufsdidaktische Perspektive}

Tobias Kärner, Hannes Reinke, Anja Frim und Karin Heinrichs Innere Differenzierung im Unterricht mit jugendlichen Asylsuchenden und Geflüchteten aus der Sicht von Lehrpersonen

Bärbel Wesselborg, Ulrike Weyland und Marc Kleinknecht

Entwicklung eines fachdidaktischen Kategoriensystems zur Analyse des kognitiv-aktivierenden Potenzials von Aufgaben - ein Beitrag zur Unterrichtsqualitätsforschung in der beruflichen Fachrichtung Pflege

Julia Schultheis und Carmela Aprea

Entwicklung und Validierung eines Schemas zur Evaluation von Serious

Games im Kontext von Financial Literacy

Tim Stanik

Mikrodidaktische Planungen von Trainerinnen und Trainern in der betrieblichen Weiterbildung 


\title{
Innere Differenzierung im Unterricht mit jugendlichen Asylsuchenden und Geflüchteten aus der Sicht von Lehrpersonen
}

\author{
Tobias Kärner, Hannes Reinke, Anja Frim und Karin Heinrichs
}

\section{Ausgangslage und Zielstellung}

Im Zuge anhaltender Flucht- und Migrationsbewegungen aus unterschiedlichen Regionen der Erde finden auch zahlreiche Jugendliche und junge Erwachsene den Weg nach Deutschland und somit in das deutsche Bildungssystem. Im Alter von 16 bis 25 Jahren stellen sie dabei einen Anteil von etwa 20 bis $30 \%$ der Asylerstanträge in den vergangenen Jahren und repräsentieren somit eine der größten Altersgruppen (BAMF 2018). Im Sinne einer erfolgreichen Integration gilt es daher vor allem auch bildungspolitisch, den damit einhergehenden Herausforderungen zu begegnen. Lange Zeit standen junge Asylsuchende und Geflüchtete $(\mathrm{jAuG})$ hierzulande nicht im Fokus entsprechender Maßnahmen. Laut Berthold (2014) liegt dies u. a. daran, dass es sich bei ihnen bisher um eine relativ kleine Gruppe im Vergleich zur Gesamtzahl aller Schüler*innen (SuS) in Deutschland handelt. Doch spätestens die veränderte Situation seit der Fluchtwelle im Jahr 2015 verdeutlicht die Notwendigkeit des Umdenkens. In diesem Kontext gilt es auch zu beachten, dass der Anteil der SuS mit Migrationshintergrund bereits vor Jahrzehnten angestiegen ist. Beispielsweise brachte der Mikrozensus aus dem Jahr 2013 hervor, dass beinahe $30 \%$ der SuS in Deutschland einen Migrationshintergrund haben (StBA 2014). In Abhängigkeit vom Einzugsgebiet stellen sie, gerade in jüngeren Jahrgängen, sogar eine Mehrheit der Schülerschaft dar (Leiprecht \& Steinbach 2015). Dies zeigt einerseits, dass es sich bei SuS mit Migrationshintergrund keineswegs um eine vernachlässigbare Minderheit handelt. Andererseits weisen die Ergebnisse internationaler Vergleichsstudien, wie beispielsweise PISA (Baumert et al. 2001), auf eine Bildungsbenachteiligung der genannten Personengruppe hin. Vergleichbare Ergebnisse können nach Behrensen und Westphal (2009) auch für $\mathrm{jAuG}$ angenommen werden.

Es ist zu berücksichtigen, dass sich Klassen mit Geflüchteten hinsichtlich ihrer Zusammensetzung durch einen hohen Grad an Heterogenität auszeichnen, da sich neben interindividuellen Unterschieden in Herkunftsländern, Konfessionen und Muttersprachen vor allem große Differenzen in den jeweiligen schulischen Vorkenntnissen und im Sprachniveau zeigen (z. B. Maué, Schumann \& Diehl 2018). Die individuellen Lernvoraussetzungen streuen hierbei 
breit: von Lernenden, welchen eine grundlegende Alphabetisierung fehlt bis zu SuS, welche in ihrem Herkunftsland bereits eine landesspezifisch gute schulische Ausbildung erhalten haben. Der Umgang mit leistungsbezogener, kultureller, religiöser und sprachlicher Heterogenität im Klassenzimmer stellt für die eingesetzten Lehrpersonen eine große Herausforderung dar (Kärner, Feldmann, Heinrichs, Neubauer \& Sembill 2016). Die Lehrkräfte sind mit der Aufgabe konfrontiert, möglichst alle Lernenden zu Handlungsfähigkeit und Berufsreife zu führen und gleichzeitig den spezifischen Bedürfnissen des/der Einzelnen gerecht zu werden (Witte \& Harden 2010). Um mit der betreffenden Schülerschaft umzugehen, wird der Einsatz von Methoden und Maßnahmen der inneren Differenzierung (i. D.) gefordert (z. B. Zoyke 2017). So soll den individuellen Bedürfnissen der Lernenden möglichst gut nachgekommen und somit zu einer gelingenden beruflichen, schulischen und gesellschaftlichen Integration beigetragen werden (vgl. Heinrichs et al. 2016). Welche konkreten Maßnahmen der Binnendifferenzierung im Unterricht mit jAuG jedoch als sinnvoll und praktikabel erscheinen und welche Kompetenzen und Eigenschaften zur Umsetzung entsprechender Maßnahmen aus Sicht der Lehrenden als notwendig und relevant eingeschätzt werden, erscheint bislang nicht hinlänglich erforscht zu sein. Hier setzt der vorliegende Beitrag an. Im Rahmen einer explorativen Interviewstudie soll den folgenden Forschungsfragen nachgegangen werden:

1. Auf welche unterrichtsbezogenen Aspekte zielen die im Rahmen der Beschulung von jAuG eingesetzten Methoden der i. D. ab und welche Schüler*innenmerkmale werden als Differenzierungskriterien herangezogen?

2. Welche Grenzen bzw. Barrieren sehen Lehrkräfte bei der Umsetzung von Methoden der i. D. in sog. Flüchtlingsklassen?

3. Inwiefern halten sich die Lehrkräfte für fähig bzw. ausreichend ausgebildet, Methoden der i. D. im Unterricht mit jAuG adäquat anzuwenden und welche Fortbildungsmaßnahmen sehen sie als erforderlich an?

Es wurden insgesamt 16 Lehrpersonen, welche in Flüchtlingsklassen in der Sekundarstufe unterrichten, mittels Leitfrageninterviews befragt. Die Aussagen wurden anschließend inhaltsanalytisch ausgewertet. Nachfolgend werden generelle bildungspolitische Bedingungen zur Beschulung von $\mathrm{jAuG}$ im deutschen Bildungssystem sowie Potenziale und Herausforderungen der i. D. im Rahmen der Flüchtlingsbeschulung diskutiert. 


\section{Hintergrund}

\subsection{Jugendliche Asylsuchende und Geflüchtete im deutschen Bildungssystem}

Seit dem Jahr 2010 haben nahezu zwei Millionen Menschen Asyl in Deutschland gesucht, wobei die meisten Anträge im Zeitraum von 2015 bis 2017 gestellt wurden. Eine der zahlenmäßig stärksten Gruppen unter den Erstantragsstellern stellen Jugendliche und junge Erwachsene im Alter von 16 bis 25 Jahren dar, die etwa 20 bis $30 \%$ der jährlichen Kohorten ausmachen und als besonders (aus-)bildungsrelevant gelten (BAMF 2018). Jäger (2002) kritisiert in diesem Zusammenhang, dass die Mehrzahl der Zielländer der in verschiedenen Abkommen festgehaltenen Aufgabe, Geflüchteten am gesellschaftlichen und wirtschaftlichen Leben teilhaben zu lassen, nicht gerecht werden und keine angemessene Förderung umsetzen. Der Stellenwert von Bildungsangeboten werde im Fall von jAuG häufig als nachrangig betrachtet (ebd.), obwohl Bildung für eine erfolgreiche Integration maßgeblich ist (Berthold 2014).

In Deutschland ist man um eine Umsetzung entsprechender internationaler Abkommen und Verträge bemüht (z. B. UNGA 1948). JAuG werden in zahlreichen, bundeslandspezifisch konzipierten Initiativen und Maßnahmen schulisch qualifiziert und auf eine Ausbildung vorbereitet. Ziel der verschiedenen Maßnahmen ist es hierbei, durch Bildung und Qualifikation eine gelingende Integration in die deutsche Gesellschaft zu ermöglichen (ISB 2017). In Bayern bspw. wurden großflächig sogenannte Berufsintegrationsklassen implementiert (für eine aktuelle Analyse zu jungen Geflüchteten in der Berufsvorbereitung in Baden-Württemberg siehe Maué et al. 2018). Asylsuchende und Geflüchtete haben die Möglichkeit vom 17ten bis zum 25sten Lebensjahr die Berufsschule zu besuchen und sich wichtige Kompetenzen ,für eine erfolgreiche Berufsausbildung und gelingende Integration“ (ISB 2017, 4) anzueignen. Das Konzept der Berufsintegrationsklassen sieht insgesamt zwei jeweils einjährige Phasen der Beschulung vor. In der Vorklasse liegt der Fokus v. a. auf der Alphabetisierung und dem Spracherwerb. Im zweiten Jahr werden neben der weiterführenden Sprachförderung v. a. berufsvorbereitende Aspekte thematisiert, welche optimaler Weise von geeigneten Betriebspraktika zur Berufsorientierung begleitet werden (hierzu Reinke, Kärner \& Heinrichs 2018). Der Besuch der zwei Schuljahre schließt mit dem Erwerb der Berechtigungen eines Mittelschulabschlusses ab (ISB 2017). 


\subsection{Innere Differenzierung im Rahmen der Flüchtlingsbeschulung}

\subsubsection{Begriffsverständnis}

Da es sich bei den $\mathrm{jAuG}$ in den betreffenden Klassen um eine als heterogen wahrgenommene Gruppe handelt (z. B. Heinrichs et al. 2016; Kärner et al. 2016; Schumann, Kärner \& Maué 2018), macht dies in besonderer Weise eine differenzierte und auf die Lernenden angepasste Unterrichtsgestaltung erforderlich. Von i. D. bzw. Binnendifferenzierung spricht man im schulischen Kontext, wenn es um „Unterscheidung, Verfeinerung, Abstufung und Aufteilung der Lerninhalte" (Paradies \& Linser 2013, 10) sowie um die Adaption der Lehrmethode, des Lerngegenstands und des Medieneinsatzes an die individuellen Lernvoraussetzungen der SuS geht. Bönsch (2016) definiert i. D. als alle Maßnahmen, „die innerhalb fester Lerngruppen (Klassen) Lernwege, Lernaufgaben, Lernansprüche, Lernzeiten und Lernhilfen für einzelne Lerner oder mindestens für Teilgruppen variabel und damit unterschiedlich gestalten." (208f.). Hierbei wird das Ziel einer möglichst individualisierten Förderung angestrebt (Kranert \& Stein, in Druck). Die i. D. ist abzugrenzen von der äußeren Differenzierung, bei welcher anhand von Selektionsmaßnahmen (z. B. Jahrgangsklassen, Schulformen) versucht wird, möglichst homogene Personengruppen zu bilden, welche räumlich getrennt voneinander unterrichtet werden (Scholz 2010). Für eine erfolgreiche Integration von Maßnahmen der i. D. in den schulischen Unterricht sind nach Klafki und Stöcker (2007) Variationen didaktischer Entscheidungsfelder hinsichtlich Aufgabenstellung (z. B. Stoffumfang, Zeitaufwand), Unterrichtsmethoden (z. B. Stationenlernen, Freiarbeit, Projektarbeit), Medien (z. B. Texte, Bilder, Computer), Sozialformen (z. B. Gruppenarbeit, Einzelarbeit) und dem Grad der Lehrerhilfe (z. B. Grad der Strukturierungshilfe) denkbar.

\subsubsection{Herausforderungen bei der Förderung von SuS mit Fluchthintergrund}

Binnendifferenzierungsmaßnahmen mit dem Zweck der individuellen Förderung werden als zielführend betrachtet, um SuS mit Migrations- bzw. Fluchthintergrund adäquat zu unterstützen (Behrensen \& Westphal 2009). Gerade in Deutschland spielt die Herkunft von Kindern und Jugendlichen für den Bildungserfolg eine zentrale Rolle. Den Ergebnissen der PISA-Studie(n) zufolge, schneiden SuS mit Migrations- bzw. Fluchthintergrund deutlich schlechter ab als SuS ohne entsprechenden Hintergrund (Kauffmann, Knapp, Novotny \& Schoch 2002). Dies hat wiederum zur Folge, dass Migranten in Förder- und Hauptschulen überrepräsentiert sind (Leiprecht \& Steinbach 2015) und hinsichtlich einer förderlichen Unterrichtsgestaltung besonderer Aufmerksamkeit bedürfen. Betrachtet man die Befundlage zur Wirksamkeit differenzierender 
Unterrichtsmaßnahmen, zeigt sich allerdings ein eher ernüchterndes Bild (vgl. Beck et al. 2008). Erklärungsversuche für die geringen Effekte von i. D. bzw. von adaptivem Unterricht setzen meist bei den hohen und komplexen Anforderungen an die Lehrpersonen an, die eine vollständige Umsetzung des Konzepts mit sich bringt. Weinert (1997) zufolge grenzt die Umsetzung an ein „wahrhaft herkulische[s] pädagogische[s] Problem“ (50), da die Gelingensbedingungen von der Motivation und dem Interesse der zu fördernden SuS bis hin zu strukturellen Bedingungen, geeigneten Räumlichkeiten, zeitlichen Ressourcen, flexiblen Budgets sowie dem Ausbildungsstand des Lehrpersonal reichen. Die gängige Praxis zeigt jedoch, dass sich diese weitreichenden Gelingensbedingungen in der Flüchtlingsbeschulung nur schwer umsetzen lassen. Barth und Guerrero Meneses (2012) kommen im Rahmen ihrer Interviewstudie zu dem Ergebnis, dass jAuG innerhalb der bestehenden Struktur von Förder- und Übergangsklassen nur sehr schwer entsprechend ihrer Fähigkeiten unterstützt werden können.

\subsubsection{Relevante Dimensionen des Lehrer*innenhandelns}

In der Diskussion um einen professionellen, ressourcen- und chancenorientierten Umgang mit Heterogenität wird insbesondere den Lehrkräften vorgeworfen, dass diese die Vielfalt ihrer SuS eher als Störgröße denn als Normalfall betrachten (Leiprecht \& Lutz 2015). Somit wird der Eindruck vermittelt, dass die Potenziale der i. D. in erster Linie deshalb eingeschränkt sind, weil die Vorbehalte (z. B. bezogen auf den zeitlichen und organisationalen Aufwand) vieler Pädagogen*innen bzw. Lehrender einer Umsetzung vorhandener Ideen entgegenstehen (Wischer \& Trautmann 2012). Die Forderung lautet demnach, dass Heterogenität von den Lehrenden idealerweise als Chance und nicht als Belastung verstanden werden soll (vgl. Terhart 2015). Wischer (2007) hingegen warnt davor, die bisherigen Defizite der Unterrichtspraxis im Umgang mit Vielfalt vorwiegend als eine Frage des Wollens zu interpretieren und dabei die Frage des Könnens außer Acht zu lassen. Wenn sich Handlungsempfehlungen ausschließlich auf die didaktisch-methodische Unterrichtsgestaltung konzentrieren, bleibt die Frage offen, inwiefern die notwendigen Voraussetzungen für deren Umsetzung - z. B. durch strukturelle Bedingungen oder durch entsprechende Ausbildungsinhalte - gegeben sind (siehe auch Trautmann \& Wischer 2013). In diesem Zusammenhang können die Befunde von Roeder (1997) angeführt werden. Diese weisen darauf hin, dass Binnendifferenzierung und individuelle Förderung von den Befragten durchaus als notwendige und wichtige Ziele wahrgenommen werden. Die befragten Lehrpersonen stehen dem Thema generell positiv gegenüber. Allerdings setzt nur eine Minderheit die erforderlichen Maßnahmen tatsächlich um. 
Da sich nach Wischer (2007) die Anforderungen an das konkrete Lehrerhandeln als sehr voraussetzungsreich darstellen, sind neben den Rahmenbedingungen auch die notwendigen Lehrer*innenkompetenzen genauer zu betrachten. Im Zuge der Diskussion bezüglich innerer Differenzierungsmaßnahmen wird hierbei auf das Konzept der adaptiven Lehrkompetenz verwiesen (z. B. Beck et al. 2008; Wischer 2007; Wischer \& Trautmann 2012). Adaptive Lehrkompetenz setzt sich hierbei aus vier Dimensionen zusammen: (1) Sachkompetenz (Beherrschen von Anforderungen des Unterrichtsinhalts), (2) diagnostischer Kompetenz (korrekte Beurteilung von Kenntnisstand, Lernfortschritten und Leistungsproblemen einzelner SuS), (3) didaktischer Kompetenz (Einsetzen unterschiedlicher Unterrichtsformen zur Erreichung unterschiedlicher pädagogischer Ziele) sowie (4) Klassenführungskompetenz (pädagogische Maßnahmen zur Steuerung, Führung und Begleitung der SuS einer Klasse) (Beck et al. 2008). Gruehn (2000) stellt vor allem die hohen Anforderungen an die methodischen und diagnostischen Kompetenzen von Lehrenden heraus. Im Bereich der didaktischen Kompetenz zeigen Forschungsergebnisse bereits der 1990er Jahre, dass Lehrpersonen eine Vielfalt von Methoden im Unterricht einsetzen (z. B. Dichanz \& Schwittmann 1999). Allerdings zeigen die bestehenden Befunde auch auf, dass erforderliche Maßnahmen nicht zwingend im Sinne der i. D. umgesetzt werden. Wischer (2007) kommt deshalb zu dem kritischen Fazit, dass sich Lehrkräfte zwar um einen abwechslungsreichen, schüleraktiven Unterricht bemühen, dabei jedoch selten differenzierte Lernwege eröffnen. Die referenzierten Befunde zeigen einerseits eine positive Einstellung der Lehrpersonen zur Thematik der i. D., andererseits jedoch eine mangelnde unterrichtspraktische Umsetzung auf. Somit scheint es sinnvoll, bereits im Rahmen konzeptioneller Überlegungen verstärkt auf die Frage des Könnens einzugehen, um die Diskrepanz zwischen der vorhandenen Einsicht über die Notwendigkeit innerer Differenzierungsmaßnahmen und der mangelnden schulpraktischen Umsetzung zu überwinden.

\section{Stichprobe und Untersuchungsmethode}

In der vorliegenden Untersuchung sollen die Sichtweisen der Lehrkräfte zu Methoden der i. D. sowie die spezifischen Bedarfe bzw. Bedingungen in den Übergangsklassen für jAuG exploriert werden. Dazu wurden Lehrpersonen aus der Sekundarstufe (Mittelschule, Berufsschule; Bayern) rekrutiert (Frim 2016). Insgesamt wurden 16 Personen (50 \% männlich) mit einem durchschnittlichen Alter von 41,7 Jahren (Min. = 31, Max. =64) befragt. 14 Personen haben ein Lehramtsstudium absolviert, zwei weitere Personen haben einen Abschluss im Fach „Deutsch als Zweitsprache“ (DaZ). Zudem besitzen elf der 
befragten Lehrkräfte eine DaZ-Zusatzqualifikation. Die Erfahrung im Unterrichten von Asylsuchenden und Geflüchteten bzw. Migranten liegt durchschnittlich bei 7,4 Jahren (Min. = 1, Max. = 25). Die 16 Lehrkräfte wurden einzeln und persönlich in halbstrukturierten Interviews befragt. Im Anschluss an die Datenerhebung wurden die Audiodaten transkribiert und mittels strukturierender Inhaltsanalyse auf Grundlage eines deduktiv-induktiven Kategorienschemas ausgewertet. ${ }^{1}$

\section{Ergebnisse}

\subsection{Unterrichtsbezogene Ansatzpunkte innerer Differenzierung}

Hinsichtlich der Frage, auf welche unterrichtsbezogenen Aspekte die im Rahmen der Beschulung von jAuG eingesetzten Methoden der i. D. abzielen, finden sich die häufigsten Nennungen zur Differenzierung anhand der Aufgabenstellung (z. B. Variation des Schwierigkeitsgrads) (15). Jeweils elf Nennungen entfallen auf die seitens der Lehrkraft getätigte unmittelbare individuelle Unterstützung bzw. Hilfestellung („Lehrerhilfe“) und auf die Sozialform (z. B. Gruppenarbeiten, Einzelarbeit). Darüber hinaus wurden Medien (z. B. Lernkarten, haptische Materialien) (5) und didaktische Methoden (z. B. Lerntheken) (2) als weitere Ansatzpunkte hervorgehoben (Abb. 1).

\section{Abb. 1: Unterrichtsbezogene Ansatzpunkte innerer Differenzierung}

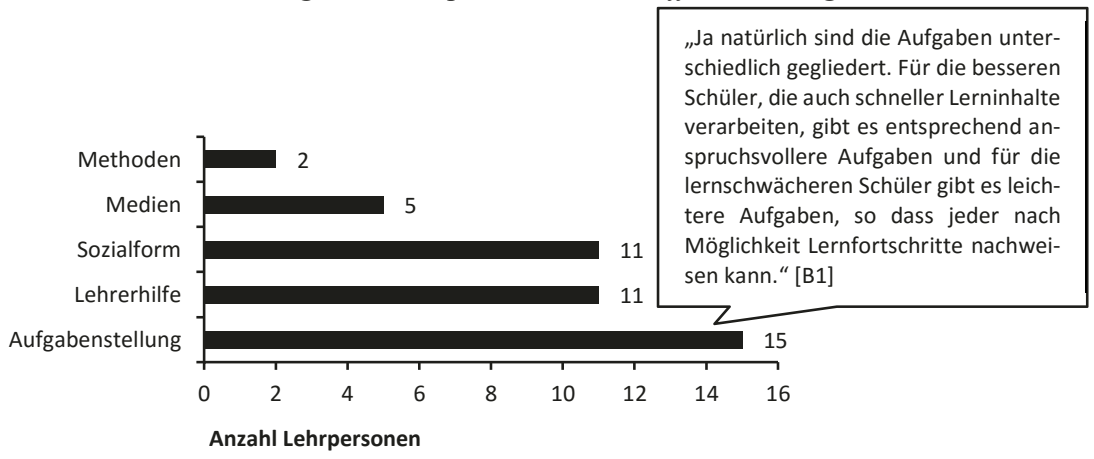

Quelle: eigene Darstellung

1 Intracoderreliabilität: Cohens Kappa-Werte von 0,85 (auf Basis von 61 doppelt kodierten Sinneinheiten) bzw. von 0,86 (74 doppelt kodierte Sinneinheiten). Intercoderreliabilität: Cohens Kappa-Werte von 0,76 (44 doppelt kodierte Sinneinheiten) bzw. 0,73 (70 doppelt kodierte Sinneinheiten). 


\subsection{Schüler*innenmerkmale als Differenzierungskriterien}

Bezogen auf die Frage, welche Schüler*innenmerkmale von den Lehrpersonen als Differenzierungskriterien herangezogen werden, orientieren sich alle befragten Lehrpersonen an dem Leistungsvermögen der Lernenden, welches meist mit den vermuteten kognitiven Kapazitäten (i. d. Grundintelligenz) gleichgesetzt wird. Die Hälfte der Teilnehmer*innen nannte das Sprachniveau in der deutschen Sprache als weiteres Differenzierungsmerkmal, gefolgt von soziodemografischen Merkmalen (z. B. Herkunft, Schichtzugehörigkeit, Sprache). Auch wurden das Interesse der Lernenden (z. B. bzgl. individueller Berufswünsche und -vorstellungen), deren Alter und das Geschlecht als mögliche Differenzierungsmerkmale genannt (Abb. 2).

Abb. 2: Schüler*innenmerkmale als Differenzierungskriterien

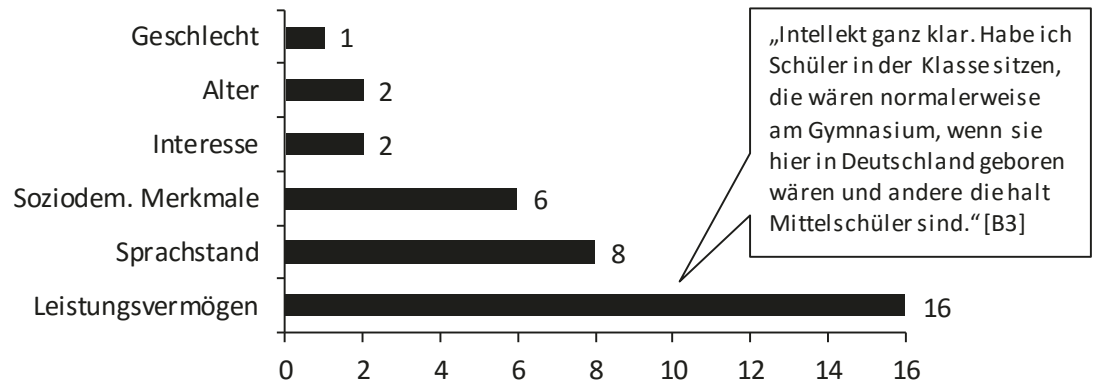

Anzahl Lehrpersonen

Quelle: eigene Darstellung

\subsection{Wahrgenommene Grenzen des Einsatzes innerer Differenzierungsmaßnahmen}

Auf die Frage nach wahrgenommenen Grenzen des Einsatzes innerer Differenzierungsmaßnahmen wurde eine ganze Bandbreite an möglichen Problemfeldern genannt. Insbesondere der zeitliche und organisatorische Mehraufwand für die Planung und den Einsatz von Binnendifferenzierungsmaßnahmen wird als sehr hoch eingeschätzt (13). Weiterhin wurden ungünstige Schüler*innenvoraussetzungen (z. B. geringe Vorkenntnisse und Fertigkeiten, psychische Beeinträchtigungen) und organisatorische Rahmenbedingungen (z. B. fehlende Räumlichkeiten und Lehr-/Lernmaterialien) von jeweils zwölf Lehrpersonen genannt. Auch sehen die Befragten in der „Heterogenität“ der Schülerschaft (11; z. B. bzgl. individueller Lernvoraussetzungen), dem Mangel an 
Lehrpersonal (10) sowie in großen Klassen (10) zusätzliche Problemfelder. Die eigene unzureichende Ausbildung im Bereich der i. D. (7), Sprachbarrieren (7), kulturelle Konflikte zwischen den Lernenden (5) sowie eine seitens der SuS wahrgenommene Ungleichbehandlung $(3$; z. B. aufgrund unterschiedlicher Zuwendungsgrade durch die Lehrperson) werden als weitere Grenzen des Einsatzes innerer Differenzierungsmaßnahmen genannt (Abb. 3).

\section{Abb. 3: Wahrgenommene Grenzen des Einsatzes innerer Differenzierungsmaßnahmen}

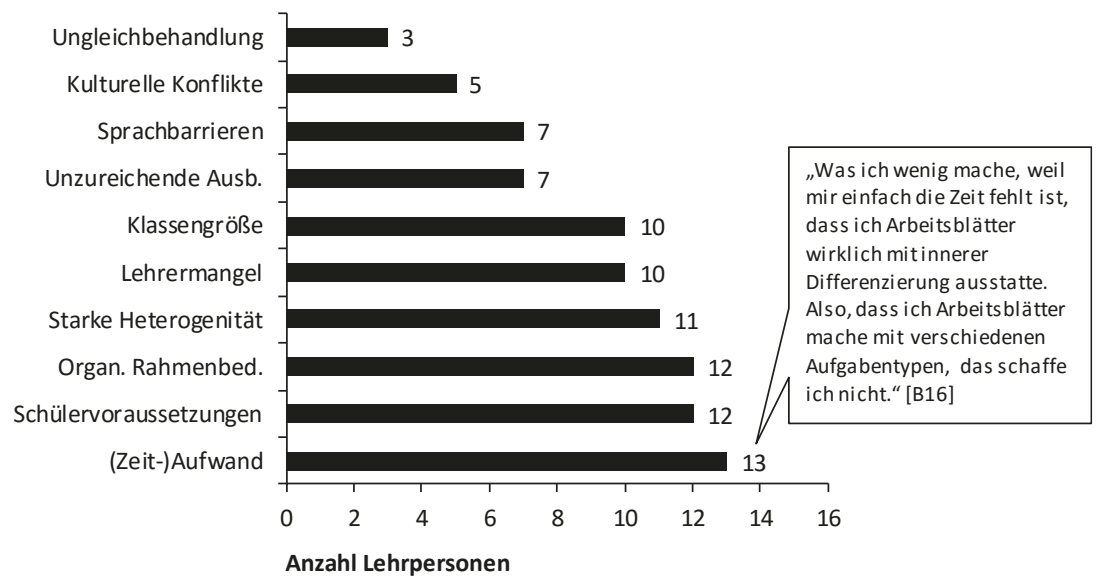

Quelle: eigene Darstellung

\subsection{Als relevant erachtete Lehrer*innenkompetenzen und -eigenschaften}

Auf die Frage nach Lehrer*innenkompetenzen und -eigenschaften, die für die Realisierung des Konzepts der i. D. im Flüchtlingsunterricht nach Ansicht der Befragten relevant sind, erwähnten zwölf von 16 Lehrpersonen interkulturelle und soziale Kompetenzen (z. B. Wissen bzgl. der Herkunftsländer), gefolgt von didaktischen (9; z. B. angemessene Aufbereitung der Lernmaterialien), diagnostischen (8; z. B. angemessene Lernstandserfassung) und Organisationskompetenzen (8; z. B. zeitliche Strukturierung des Unterrichts). Weiterhin nannten sechs der Befragten Geduld als eine wichtige Eigenschaft (z. B. aufgrund unterschiedlicher Lerngeschwindigkeiten). Jeweils zwei Lehrpersonen betonten Klassenführungs- (z. B. Anleiten von Lerngruppen) und Sachkompetenz (z. B. Fachkenntnisse bzgl. der behandelten Inhalte) (Abb. 4). 
Abb. 4: Als relevant erachtete Lehrer*innenkompetenzen und-eigenschaften

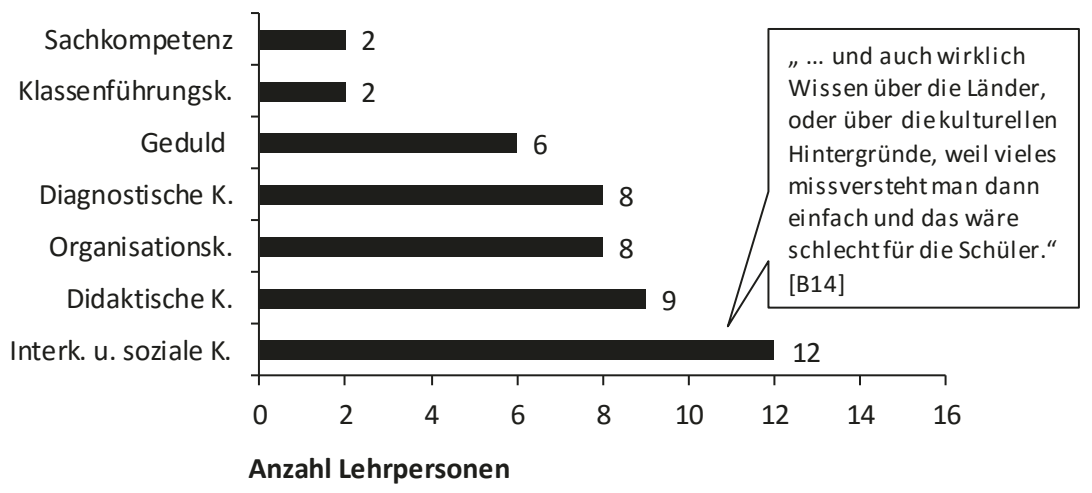

Quelle: eigene Darstellung

\subsection{Thematisierung in der Lehrer*innenbildung und Fort- und Weiterbildungsbedarfe}

Hinsichtlich der Frage, inwiefern sich die Lehrkräfte für fähig bzw. ausreichend ausgebildet halten, Methoden der i. D. im Unterricht mit jAuG adäquat anzuwenden, erachteten zwölf der befragten Lehrpersonen die Behandlung von Binnendifferenzierungsmethoden in ihrem Studium als lückenhaft und nur zwei Lehrpersonen haben den Eindruck, dass dieses Thema in ihrem Studium in ausreichendem Maße behandelt wurde. Neun von 14 Personen (zwei der Befragten haben kein Referendariat absolviert) sehen die Thematisierung von Binnendifferenzierungsmaßnahmen im Referendariat als ausreichend behandelt an. Fünf Personen dagegen vertreten die Meinung, dass in der zweiten Phase der Lehrer*innenbildung das Thema nur lückenhaft behandelt werde.

Nach notwendigen Fort- und Weiterbildungsthemen gefragt, machten zwölf Lehrpersonen deutlich, dass sie im didaktischen Bereich hinsichtlich konkreter Methoden und Instrumente der Binnendifferenzierung Bedarf sehen. Eine Lehrperson sieht für sich, trotz vorhandener DaZ-Qualifikation, Bedarf bezüglich der Vermittlung einer grundlegenden Alphabetisierung, eine andere Lehrkraft erachtet für sich eine Fortbildung im Bereich der Didaktik des Deutschen als Zweitsprache als sinnvoll und eine weitere Lehrperson sah die Notwendigkeit in Bezug auf Diagnostikverfahren hinsichtlich des individuellen Leistungsvermögens der Lernenden. 


\section{Schlussteil}

\subsection{Limitationen der Studie}

Limitierend ist bzgl. der Interviewstudie anzumerken, dass diese lediglich einen ersten explorativen Zugang zum behandelten Themenschwerpunkt darstellt und aufgrund der qualitativen Methodik sowie der kleinen Stichprobe keine (statistische) Prüfung von Hypothesen zulässt. Um insbesondere hinsichtlich der Wirkung binnendifferenzierender Maßnahmen auf lern-, leistungs- und entwicklungsrelevante Indikatoren der betrachteten Klientel belastbare Aussagen treffen zu können, sind zukünftig entsprechende experimentelle Versuchsanordnungen unumgänglich. Hierfür kann die vorliegende Studie jedoch einen orientierungsgebenden Ausgangspunkt darstellen. Weiterhin ist anzumerken, dass die Datenerhebung bereits im Jahr 2016 stattfand, was angesichts des sich schnell entwickelnden und verändernden Gegenstandsbereichs der Beschulung jAuG ebenfalls einen limitierenden Faktor darstellt.

\subsection{Von wahrgenommenen Barrieren zu Entwicklungschancen}

Das Wollen bzgl. des Einsatzes innerer Differenzierungsmaßnahmen im Unterricht mit jAuG im Sinne von Wischer (2007) scheint nach den Aussagen der von uns befragten Lehrpersonen gegeben zu sein. Auch werden bereits unterschiedliche Maßnahmen und Methoden in den Unterricht integriert, was jedoch in der schulischen Praxis an unterschiedlichen Stellen an seine Grenzen stößt - die Frage des Könnens i. S. v. Wischer (2007) ist demnach vor dem Hintergrund der gefundenen Ergebnisse zu diskutieren.

Die Etablierung individueller Förderung setzt jedoch entsprechende Rahmenbedingungen voraus. Die identifizierten Barrieren, welche nach den Aussagen der Lehrkräfte eine Umsetzung von Binnendifferenzierungsmaßnahmen im Unterrichtsalltag erschweren, reichen von allgemeinen Rahmenbedingungen bis zur Ebene der beteiligten Personen. Hierbei wird deutlich, dass eine erfolgreiche Etablierung von Beschulungsmaßnahmen für $\mathrm{jAuG}$ vermutlich nur über eine sehr enge Verzahnung von äußeren und inneren Differenzierungsmaßnahmen gelingen kann. Dies zeigte sich auch in unserer Untersuchung, wobei auf der Ebene der Lehrpersonen besonders häufig die Faktoren (Zeit-)Aufwand, organisatorische Rahmenbedingungen und Schüler*innenvoraussetzungen als kritische Punkte genannt wurden (siehe auch Heinrichs et al. 2016; Heinrichs, Reinke \& Feldmann 2018). Hinzu kommen der sog. „Lehrermangel", die Klassengrößen und spezifische Grenzen im Bereich der Flüchtlingsbeschulung wie z. B. die ,starke Heterogenität" der Schülerschaft sowie Sprachbarrieren. Zumindest bzgl. des letztgenannten Punktes wird versucht, 
mit Hilfe des Unterrichtsprinzips „Berufssprache Deutsch“ und bereitgestellter Materialien zur sprachsensiblen Unterrichtsgestaltung, Unterstützungsangebote für Lehrpersonen bereitzustellen (hierzu auch Terrasi-Haufe, Hofmann \& Sogl 2018). Die Ausführlichkeit der genannten Hindernisse stützt das Bild, nach dem es sich aus Sicht der Lehrkräfte bei der Binnendifferenzierung um ein sehr voraussetzungsreiches Konzept handelt. Die seitens der Lehrpersonen wahrgenommene Überforderung bei der praktischen Umsetzung verwundert also nicht.

Eine weitere Grenze, die im Rahmen der Untersuchung zur Sprache kam, ist die als unzureichend empfundene Ausbildung bezüglich der Thematik der inneren Differenzierung. Dabei sehen die Befragten vorwiegend für die erste Phase der Lehramtsausbildung an den Hochschulen starken Nachholbedarf. Zwar gaben die meisten Befragten an, dass das Konzept der Binnendifferenzierung während des Studiums thematisiert wurde, erachten die als berufsalltagsfern wahrgenommenen Ausführungen jedoch nicht für ausreichend und zielführend. Aus diesem Grund ist über eine stärkere Kooperation der Universitäten und der Schulen bereits in der ersten Phase der Lehramtsausbildung nachzudenken, wie dies beispielsweise im Zuge der bayerischen Universitätsschulinitiative verfolgt wird (z. B. Bauer, Blum \& Sembill 2012).

\subsection{Ausblick: Passungsverhältnis zwischen Lernenden und Lernkontext}

Die Ergebnisse zeichnen ein Bild, nach dem alle der 16 befragten Lehrkräfte den Einsatz innerer Differenzierungsmaßnahmen im Unterricht mit jAuG grundsätzlich für notwendig und sinnvoll erachten. Sie sehen sich jedoch angesichts der als ,,sehr heterogen“ wahrgenommenen Schülerschaft vor der Herausforderung, ihren Unterricht adressatengerecht zu gestalten und Lernangebote $\mathrm{zu}$ schaffen, welche allen Lernenden möglichst gut gerecht werden. Hierzu erscheint es nicht ausreichend, gelegentliche, summative Lernstandsdiagnosen zum Zwecke der Notenfindung durchzuführen. Vielmehr müssen die Lehrpersonen, um Lernverläufe nachvollziehen und adäquat begleiten zu können, im Unterrichtsprozess permanent diagnostische Informationen aufnehmen und verarbeiten, was in der unterrichtlichen Praxis i. d. R. beiläufig und routinebasiert geschieht. Die lehrer*innenseitige Verarbeitung von Informationen als Grundlage bedarfsgerechter und situationsangemessener didaktischer Entscheidungen ist hierbei jedoch $u$. a. aufgrund von Informationsflut und -menge sowie beschränkter Beobachtbarkeit schüler- und unterrichtsseitiger Lernindikatoren sehr anfällig für Urteilsfehler und damit einhergehende Fehleinschätzungen (Warwas, Kärner \& Golyszny 2015). Angesichts ,heterogener" Lernvoraussetzungen und -bedingungen sind hierbei neben interindividuellen Unterschieden zwischen Lernenden (z. B. individuelles Vorwissen zu Beginn eines neuen Unterrichtsthemas), ebenso intraindividuelle Veränderungen 
(z. B. zeitpunktbezogene Fachwissensniveaus) sowie Variationen im Zusammenspiel zwischen den Lernenden und dem Lernkontext (z. B. Interaktionen zwischen individuellen Fachwissensniveaus und situativen Aufgabenschwierigkeiten) zu bedenken (Kärner, Warwas \& Schumann 2019). Dieses komplexe Interaktionsgefüge macht es insbesondere vor dem Hintergrund der Fragestellung nach einer möglichst optimalen Passung zwischen Differenzierungsmaßnahmen und schüler*innenseitigen Differenzierungskriterien erforderlich, Lehrpersonen durch Möglichkeiten der gezielten Analyse und Aufbereitung von inhalts- und schülerbezogenen Informationen beim Treffen begründeter pädagogisch-diagnostischer Entscheidungen zu unterstützen, was u. a. durch entsprechende Informationssysteme erreicht werden kann (z. B. Kärner, Fenzl, Warwas \& Schumann 2019; Kärner, Warwas \& Schumann 2019).

\section{Literatur}

BAMF (Bundesamt für Migration und Flüchtlinge). (2018). Aktuelle Zahlen zu Asyl (11/2018). Online: http://www.bamf.de/DE/Infothek/Statistiken/Asylzahlen/AktuelleZahlen/aktuelle-zahlen-asyl-node.html (15.01.2019).

Barth, S. \& Guerrero Meneses, V. (2012). Zugang jugendlicher Asylsuchender zu formellen Bildungssystemen in Deutschland. Zwischen Kompetenzen und strukturellen Problemlagen. Online: https://isis-sozialforschung.de/docs/Bildungszug $\% \mathrm{c} 3 \% \mathrm{a} 4$ ngef\%c3\%bcrAsylsuchende.pdf (06.05.2016).

Bauer, C., Blum, E. \& Sembill, D. (2012). Bamberger Universitätsschulen: Sicherung von Qualität und Quantität der Lehrerbildung in der beruflichen Bildung. Wirtschaft \& Erziehung, 64(7), 218-220.

Baumert, J., Klieme, E., Neubrand, M., Prenzel, M., Schiefele, U., Schneider, W., Stanat, P., Tillmann, K.-J. \& Weiß, M. (Hrsg.). (2001). PISA 2000. Basiskompetenzen von Schülerinnen und Schülern im internationalen Vergleich. Opladen: Leske + Budrich.

Beck, E., Baer, M., Guldimann, T., Bischoff, S., Brühwiler, C., Müller, P., Niedermann, R., Rogalla, M. \& Vogt, F. (Hrsg.). (2008). Adaptive Lehrkompetenz. Analyse und Struktur, Veränderbarkeit und Wirkung handlungssteuernden Lehrerwissens. Münster: Waxmann.

Behrensen, B. \& Westphal, M. (2009). Junge Flüchtlinge - ein blinder Fleck in der Migrations- und Bildungsforschung. Bildung junger Flüchtlinge als Randthema in der migrationspolitischen Diskussion. In L. Krappmann, A. Lob-Hüdepohl, A. Bohmeyer \& S. Kurzke-Maasmeier (Hrsg.), Bildung für junge Flüchtlinge - ein Menschenrecht. Erfahrungen, Grundlagen und Perspektiven, (S. 45-58). Bielefeld: Bertelsmann.

Berthold, T. (2014). In erster Linie Kinder. Flüchtlingskinder in Deutschland. 2014. Online: https://www.unicef.de/blob/56282/fa13c2eefcd41dfca5d89d44c72e72e3* fluechtlingskinder-in-deutschland-unicef-studie-2014-data.pdf (05.05.2016). 
Bönsch, M. (2016). Heterogenität verlangt Differenzierung. Die berufsbildende Schule, $68(6), 208-212$.

Dichanz, H. \& Schwittmann, D. (1999). Das Methodenrepertoire von Lehrern. Eine Unterrichtsvariable im Brennpunkt. In U. Steffens \& T. Bargel (Hrsg.), Lehren und Lernen im offenen Unterricht. Empirische Befunde und kritische Anmerkungen. Beiträge aus dem Arbeitskreis „Qualität der Schule“, (S. 12-17). Wiesbaden: HELP.

Frim, A. (2016). Analysen zur inneren Differenzierung im Bereich Flüchtlingsbeschulung mittels Lehrerbefragung. Unveröffentlichte Masterarbeit an der Professur für Wirtschaftspädagogik (Prof. Dr. K. Heinrichs), Otto-Friedrich-Universität Bamberg.

Gruehn, S. (Hrsg.). (2000). Unterricht und schulisches Lernen. Schüler als Quellen der Unterrichtsbeschreibung. Münster: Waxmann.

Heinrichs, K., Kärner, T., Ziegler, S., Feldmann, A., Reinke, H. \& Neubauer, J. (2016). Die Implementierung neuer Konzepte zur Beschulung von Flüchtlingen und Asylsuchenden. Herausforderungen und Chancen aus organisationstheoretischer Perspektive. Gruppe. Interaktion. Organisation. Zeitschrift für Angewandte Organisationspsychologie, 47(3), 231-241.

Heinrichs, K., Reinke, H. \& Feldmann, A. (2018). Herausforderungen und Chancen in der Beschulung von Geflüchteten in Berufsintegrationsklassen aus Sicht der Schulen und Verwaltung 2015 und heute - eine Zwischenbilanz. Vlb-Akzente, 1/2018, $15-18$.

ISB (Staatsinstitut für Schulqualität und Bildungsforschung). (2017). Lehrplan für die Berufsintegrations- und Sprachintensivklassen. Online: https://www.isb.bayern.de /download/19735/lp_berufsintegrationsklassen_07_2017.pdf (15.01.2019).

Jäger, T. (2002). They don't need no education? Flüchtlinge und Bildung. Zeitschrift für internationale Bildungsforschung und Entwicklungspädagogik, 25(2), 2-9.

Kärner, T., Feldmann, A., Heinrichs, K., Neubauer, J. \& Sembill, D. (2016). Herausforderungen bei der Beschulung von Asylsuchenden und Flüchtlingen im Rahmen von BAF-Klassen an beruflichen Schulen: Deskriptive Befunde aus einer Interviewstudie. Wirtschaft \& Erziehung, 68(2), 58-62.

Kärner, T., Fenzl, H., Warwas, J. \& Schumann, S. (2019). Digitale Systeme zur Unterstützung von Lehrpersonen - Eine kategoriengeleitete Sichtung generischer und anwendungsspezifischer Systemfunktionen. Zeitschrift für Berufs- und Wirtschaftspädagogik, 115(1), 39-65.

Kärner, T., Warwas, J. \& Schumann, S. (2019). Addressing individual differences in the vocational classroom: towards a Teachers' Diagnostic Support System (TDSS). In T. Deißinger, U. Hauschildt, P. Gonon \& S. Fischer (eds.), Contemporary Apprenticeship Reforms and Reconfigurations, (pp. 179-182). Zürich: Lit.

Kauffmann, H., Knapp, A., Novotny, E. \& Schoch, S. (2002). Flüchtlinge und Schule? Erfahrungen. Zeitschrift für internationale Bildungsforschung und Entwicklungspädagogik, 25(2), 15-19.

Klafki, W. \& Stöcker, H. (2007). Innere Differenzierung des Unterrichts. In W. Klafki (Hrsg.), Neue Studien zur Bildungstheorie und Didaktik. Zeitgemäße Allgemeinbildung und kritisch-konstruktive Didaktik, (S. 173-208). Weinheim: Beltz. 
Kranert, H.-W. \& Stein, R. (im Druck). Multiprofessionalität in der inklusiven Unterrichtsentwicklung - Mögliche Beiträge der Sonderpädagogik in einem interdisziplinären Team an Berufsschulen. In K. Heinrichs \& H. Reinke (Hrsg.), Heterogenität in der beruflichen Bildung im Spannungsfeld zwischen Erziehung, Förderung und Fachausbildung. Reihe Wirtschaft - Beruf - Ethik. Bielefeld: wbv.

Leiprecht, R. \& Lutz, H. (2015). Intersektionalität im Klassenzimmer. Zur sozialen Konstruktion und Bedeutung von Ethnie, Klasse, Geschlecht und ihren Verbindungen. In R. Leiprecht \& A. Steinbach (Hrsg.), Schule in der Migrationsgesellschaft. Ein Handbuch. Band 1: Grundlagen - Diversität-Fachdidaktiken, (S. 283-304). Schwalbach am Taunus: Debus.

Leiprecht, R. \& Steinbach, A. (2015). Einleitung. Schule in der Migrationsgesellschaft. In R. Leiprecht \& A. Steinbach (Hrsg.), Schule in der Migrationsgesellschaft. Ein Handbuch. Band 1: Grundlagen - Diversität-Fachdidaktiken, (S. 7-22). Schwalbach am Taunus: Debus.

Maué, E., Schumann, S. \& Diehl, C. (2018). Bildungshintergrund und Bildungspläne geflüchteter Jugendlicher im System der beruflichen Bildung. In E. Wittmann, D. Frommberger \& B. Ziegler (Hrsg.), Jahrbuch der berufs- und wirtschaftspädagogischen Forschung 2018. Schriftenreihe der Sektion Berufs- und Wirtschaftspädagogik der Deutschen Gesellschaft für Erziehungswissenschaft (DGfE), (S. 137148). Berlin: Budrich.

Paradies, L. \& Linser, H. J. (Hrsg.). (2013). Differenzieren im Unterricht. Berlin: Cornelsen.

Reinke, H., Kärner, T. \& Heinrichs, K. (2018). Analyse lern- und entwicklungsförderlicher Gestaltungsbedingungen beruflicher Praktika für berufsschulpflichtige Asylsuchende und Flüchtlinge: Befunde einer Tagebuchstudie. Unterrichtswissenschaft, 46(1), 43-60.

Roeder, P. M. (1997). Binnendifferenzierung im Urteil von Gesamtschullehrern. Zeitschrift für Pädagogik, 43(2), 241-259.

Scholz, I. (2010). Pädagogische Differenzierung. Stuttgart: UTB.

Schumann, S., Kärner, T. \& Maué, E. (2018). Integration von jungen Geflüchteten in das deutsche Berufsbildungssystem: Klassenkompositionseffekte und binnendifferenzierende Maßnahmen. In M. Pilz, K. Breuing \& S. Schumann (Hrsg.), Berufsbildung zwischen Tradition und Moderne. Festschrift für Thomas Deißinger zum 60. Geburtstag, (S. 83-101). Wiesbaden: Springer VS.

StBA (Statistisches Bundesamt). (2014). Bevölkerung und Erwerbstätigkeit. Bevölkerung mit Migrationshintergrund - Ergebnisse des Mikrozensus. Online: https://www.destatis.de/DE/Publikationen/Thematisch/Bevoelkerung/MigrationIntegration/Migrationshintergrund2010220137004.pdf?_ blob=publicationFile (05.05.2016).

Terhart, E. (2015). Umgang mit Heterogenität: Anforderungen an Professionalisierungsprozesse. In C. Fischer (Hrsg.). (Keine) Angst vor Inklusion, (S. 63-83). Münster: Waxmann.

Terrasi-Haufe, E., Hofmann, M. \& Sogl, P. (2018). Sprachförderung in der beruflichen Bildung nach dem Unterrichtskonzept „Berufssprache Deutsch“. Zeitschrift für Interkulturellen Fremdsprachenunterricht, 23(1), 3-16.

Trautmann, M. \& Wischer, B. (2013). Schultheoretische Anfragen zum pädagogischnormativen Heterogenitätsdiskurs. Schweizerische Zeitschrift für Bildungswissenschaften, 35(1), 21-36. 
UNGA (United Nations General Assembly/Generalversammlung der Vereinten Nationen). (1948). Resolution der Generalversammlung 217 A (III). Allgemeine Erklärung der Menschenrechte. Online: http:/www.un.org/depts/german/menschenrechte/aemr.pdf (06.05.2016).

Warwas, J., Kärner, T. \& Golyszny, K. (2015). Diagnostische Sensibilität von Lehrpersonen im Berufsschulunterricht: Explorative Prozessanalysen mittels ContinuousState-Sampling. Zeitschrift für Berufs- und Wirtschaftspädagogik, 111(3), 437454.

Weinert, F. E. (1997). Notwendige Methodenvielfalt. Unterschiedliche Lernfähigkeit erfordert variable Unterrichtsmethoden. In M. A. Meyer, U. Rampillon, G. Otto \& E. Terhart (Hrsg.), Lernmethoden-Lehrmethoden. Wege zur Selbstständigkeit, (S. 50-53). Seelze: Friedrich.

Wischer, B. (2007). Wie sollen LehrerInnen mit Heterogenität umgehen? Über, ,programmatische“ Fallen im aktuelle Reformdiskurs. Die deutsche Schule, 99(4), 422-433.

Wischer, B. \& Trautmann, M. (2012). Innere Differenzierung als reformerischer Hoffnungsträger. Eine einführende Problemskizze zu Leerstellen und ungelösten Fragen. In T. Bohl, M. Bönsch, M. Trautmann \& B. Wischer (Hrsg.), Binnendifferenzierung. Teil 1: Didaktische Grundlagen und Forschungsergebnisse zur Binnendifferenzierung im Unterricht. Theorie und Praxis der Schulpädagogik, (S. 24-39). Immenhausen bei Kassel: Prolog.

Witte, A. \& Harden, T. (2010). Die Rolle des Lehrers/der Lehrerin im Unterricht des Deutschen als Zweit- und Fremdsprache. In H.-J. Krumm, C. Fandrych, B. Hufeisen \& C. Riemer (Hrsg.), Deutsch als Fremd- und Zweitsprache. Ein internationales Handbuch, (S. 1324-1340). Berlin: De Gruyter.

Zoyke, A. (2017). Inklusive Didaktik (wirtschafts-)beruflicher Bildung. Berufsbildung, $71,24-26$. 